\title{
Regional Human Settlement Environmental Ethical Features of Dayi Liu's Manor in the Western Sichuan Plain
}

\author{
Ziwei Luo ${ }^{1}$, Wenfeng Xie ${ }^{1 *}$, and Guohua Wang ${ }^{2}$ \\ ${ }^{1}$ College of Landscape Architecture, Sichuan Agricultural University, No.211, Huimin Road, Wenjiang District, Chengdu, China \\ ${ }^{2}$ Sichuan Guangyuan landscaping affairs center, Guangming Apartment, Dongba Binhe North Road, Guangyuan City, China
}

\begin{abstract}
Manors are the most representative human settlements in the history of Western Sichuan, with complete regional tradition and ethical order. As a protective heritage in the revitalization and development of rural areas, manors have been recognized as construction achievements for their artistic and practical references. This paper starts from the perspective of human settlement environmental ethics, focusing on the environmental ethical order between the microcosm of the residential environment of Liu's Manor and the macrocosm of the regional pattern of the Western Sichuan Plain. The result shows that the environment characteristics of Liu's manor, under the regional settlement pattern, actually are the images of human subjective ethical choice; gathering in an ideal environment, dispersing to the origin, and the integration of classical styles. On this basis, this study proposes a means through which to realize sustainable development in rural settlement construction in the process of modernization in China.
\end{abstract}

\section{Introduction}

In ancient China, early settlements built for feudal lords were called manors. Manors evolved from folk houses, it represented the highest achievement in the development of traditional residences. After the Qin and Han Dynasties, manors gradually developed into 'landlord manors' [1]. From the perspective of social influence, the landlord manor was an entity. It represents the landlord class' oppression and exploitation of the peasant. From the perspective of material environment construction, the manor often represents a superior form of local microcosmic living environment. Compared with other traditional residences, manors pay more attention to the creation of regional traditions and ethical orders. This paper takes Dayi Liu's Manor as the object, which is the largest landlord manor on the Western Sichuan Plain and the typical model of historiographical research, aiming to interpret the relationship between residential and regional environments from the perspective of ethics, and further providing a reference to the regional construction of residential settlements in the new era.

\section{Human settlement environmental ethics}

Ethics located in philosophy. It aims to understand orders and norms that people believe in for interpersonal communication. Since the 20th century, in order to solve increasingly environmental problems, people have begun to care about the stakes between human beings and the natural environment, resulting in the development of the field of environmental ethics [2]. On this basis, an ethical perspective of the human settlement environment has been further developed to study the relationship between human beings and the living environment. The purpose of applying ethical philosophy to the science of human settlements is to extend moral concern to living spaces and the natural environment and to establish a new moral and ethical order as well as a tacit understanding to guide construction planning in an environmentally conscious direction $[3,4]$.

Human settlement environmental ethics offers a concrete description of the human settlement environment. People's subjective demands for residence and subject cognition of the environment form the subject-oriented ethical choices, so as to coordinate the placement order between the microcosm residence environment image and the macrocosm regional pattern, and ensure the harmonious coexistence between the human and the environment (Figure 1).

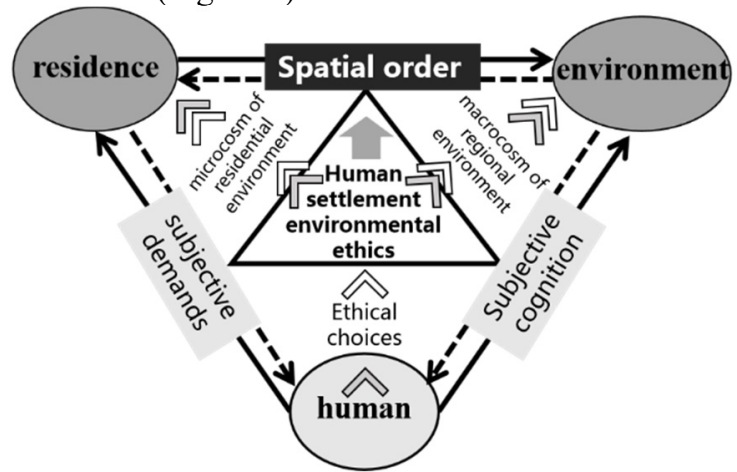

Fig1. The ethical relationship between human beings, residence, and the environment.

Manors as a kind of human settlements, developed

* Corresponding author: xwenfeng@sicau.edu.cn 
with a certain economic basis and artistic integration, are usually a combination of regional characteristics and other human cultures. They are usually built spontaneously by local people when their economic conditions are relatively good. Such buildings not only depict the original environment of the local users, but also reflect their views on environmental selection in pursuit of culture and art. The environmental image presented by the architecture is the embodiment of its ethical characteristics, especially in the area where the culture is diversified and blended. In Western Sichuan, studying the construction of human settlements through the residences represented by Liu's Manor offers insight into the historical experience of those who built, owned, and lived in the manor. The spatial order between residence and environment, formed by the cognition and the integration of the regional environmental culture, is a portrayal of the ethics of human settlements in historical heritage $[5,6]$.

\section{Macro-settlement patterns of the Western Sichuan Plain}

With Chengdu as its center and including the vast surrounding rural areas, the Western Sichuan Plain is an agricultural settlement area that integrates living and production (Figure 2). The excellent farming conditions have made it the main settlement area for immigrants from other provinces, and the settlement pattern has gradually recovered and developed steadily [7].

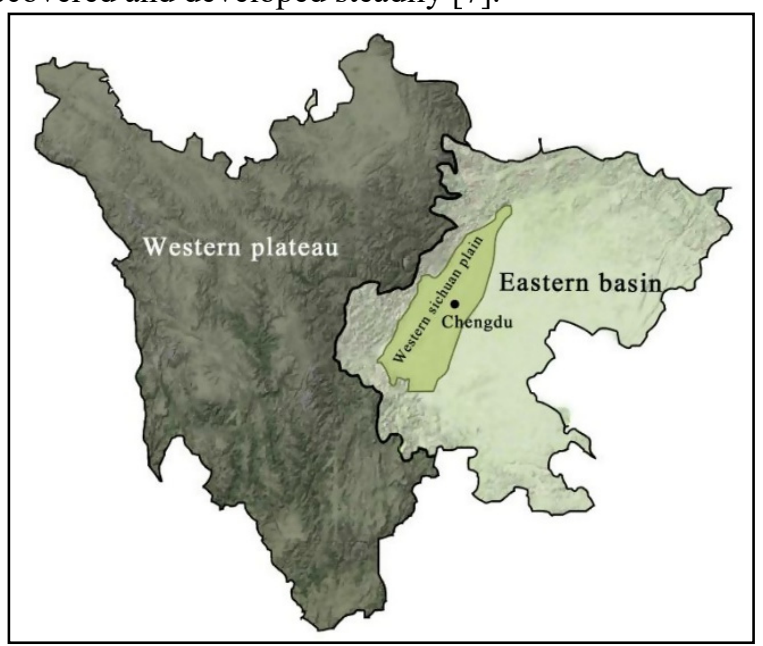

Fig2. The location of the Western Sichuan Plain in Sichuan

$$
\text { Province. }
$$

Immigrants who entered Sichuan at the beginning of the Qing Dynasty took the lead in the land reclamation, preparing fields for crops and building houses, thereby forming a settlement pattern with clans as the core community. By the middle of the Qing Dynasty, the increase in population led to a gradual saturation of cultivated land. At this time, farmers had to rent land from landlords both for agricultural and residential purposes, which gradually lead a dispersion outwards from the center of the initial settlement. By the late era of the Qing Dynasty, the landlord economy had further developed, with land subleased and subdivided. The pressure of population growth against the availability of arable land eventually led to the development of smaller settlements, which increased the density and dispersion of settlements in the region (Figure 3). Against the background of migration, the dispersion and integration of rural areas became apparent, forming a stable high-density settlement pattern. This pattern tended to be consistent at the macrolevel, and formed a regional environment characterized by gather, dispersion, and integration.
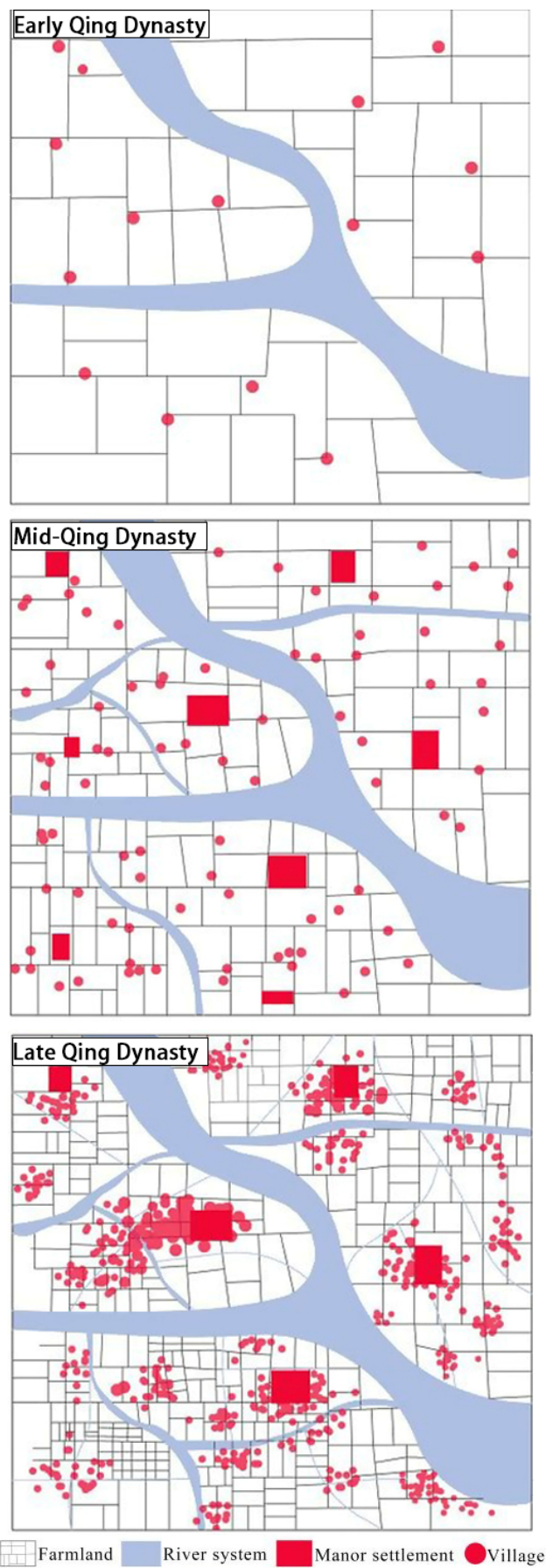

Fig3. Schematic diagram of regional environmental evolution in the Western Sichuan Plain.

\section{The environmental ethical features of Dayi Liu's Manor}

Dayi Liu's Manor is the ancestral home of Wencai Liu, a substantial landowner, whose manor is known as "the largest landlord's manor in western Sichuan". Over the 
past 100 years, the environmental features continue to reflect the owner's choices that how to harmonize the living space and the regional environment; gathering in an ideal environment, dispersing to the origin, and the integration of classical styles.

\subsection{Gathering in an ideal environment}

Liu's Manor is an advanced form of living in the history of western Sichuan's residential development. The manor reflects an environmental optimization in terms of pattern, the spatial aggregation of architecture, and identifying an ideal environment in the composition of the whole settlement.

\subsubsection{Settlement location}

Liu's Manor is located in the famous historical and cultural town of West Sichuan-Anren Ancient Town in Dayi County. In the 1930s, the construction of the mansion began. It gradually formed into the old mansion (including Liu's ancestral yard as well as Wencheng Liu, Wencai Liu, and Wenzhao Liu's house yard) and the new mansion (Wenhui Liu's house yard) (Figure 4).

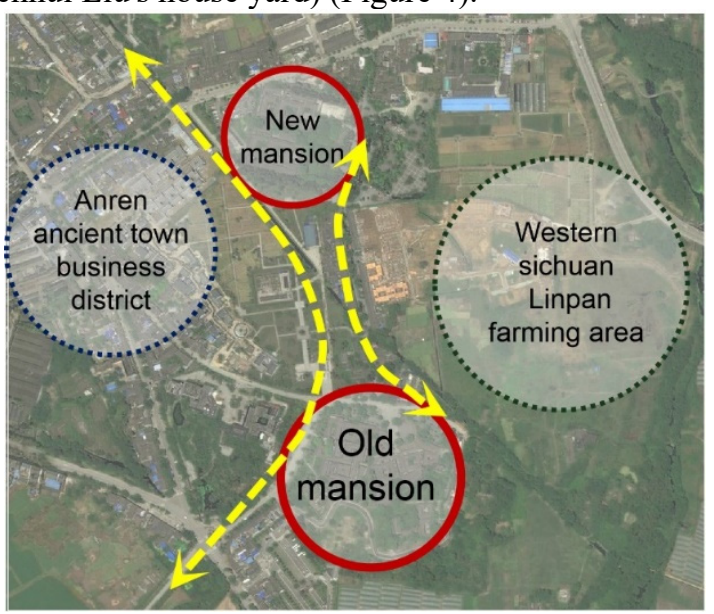

Fig4. The surrounding environment of Liu's manor.

Anren Ancient Town represents the mature model in the development of rural settlements in western Sichuan. It has distinct street spaces and architectural texture, primarily for public rallies and commercial activities, and its function is relatively holistic. In ancient times, the town was too far away from the imperial rule, and Liu's Manor was located at the intersection between the town of Anren and the vast Linpan, so that the social environment has not restricted its development. This prime location enjoyed the advantages of a perfect network system - on the edge of a natural environment, on one side, and a business and residential district, on the other-enabled the Liu family to expand the scale of their manor around the core of Liu's ancestral home using the resources of the land and relying on local transportation, markets, manpower, and other resources. From the perspective of the living environment, the manor could enjoy a vast agricultural landscape as well as the nearby commercial landscape that had gathered (Figure 5), forming a harmonious living atmosphere between gathering and dispersing. The flexible external environment supported its independent development and optimized the expression of the living space in line with the manor's status.

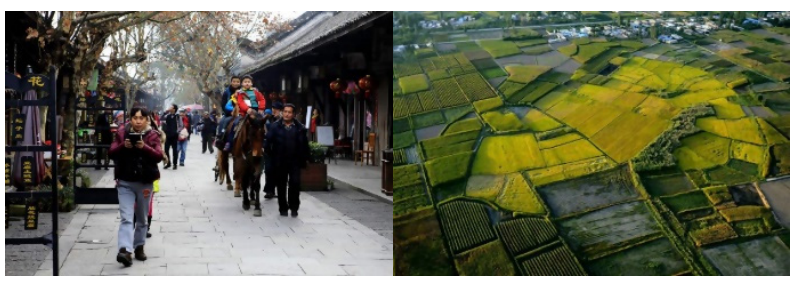

Fig5. The town and farming fields between which the manor is located.

\subsubsection{Settlement space}

Liu's Manor settlement has an undeniable spatial continuity and integrity. It has formed a very successful enclosure of woodland resources. The combination of plants and architecture is conducive to the formation of a microclimate environment, and the manor space is the greatest beneficiary of this environment.

In order to adapt to the humid, hot, and rainy climate, Liu's Manor adopted a very typical architectural systemthe Southern Siheyuan (a quadrangular courtyard)which is the highest in form of traditional dwellings. Traditional rural buildings have little space for a significant continuous courtyard space, but Liu's manor presents us with two very distinct types of continuous quadrangular courtyards: the differently sized and freeform layout of the old manor complex and the very obvious axis layout of the new manor complex (Figure 6).

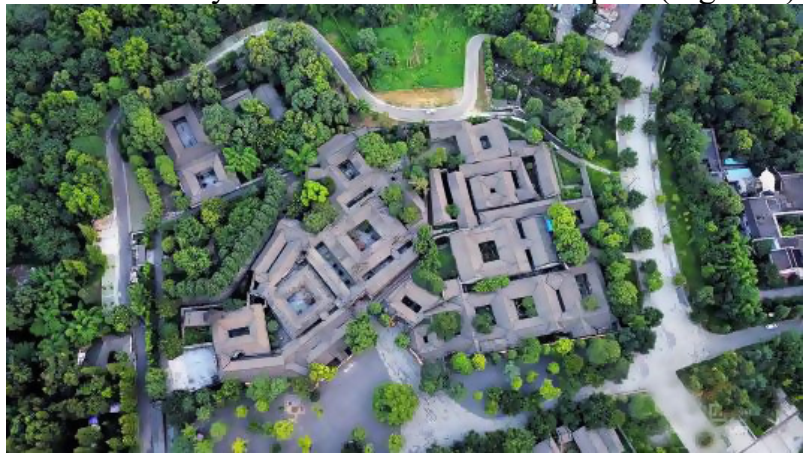

a.The old manor complex.

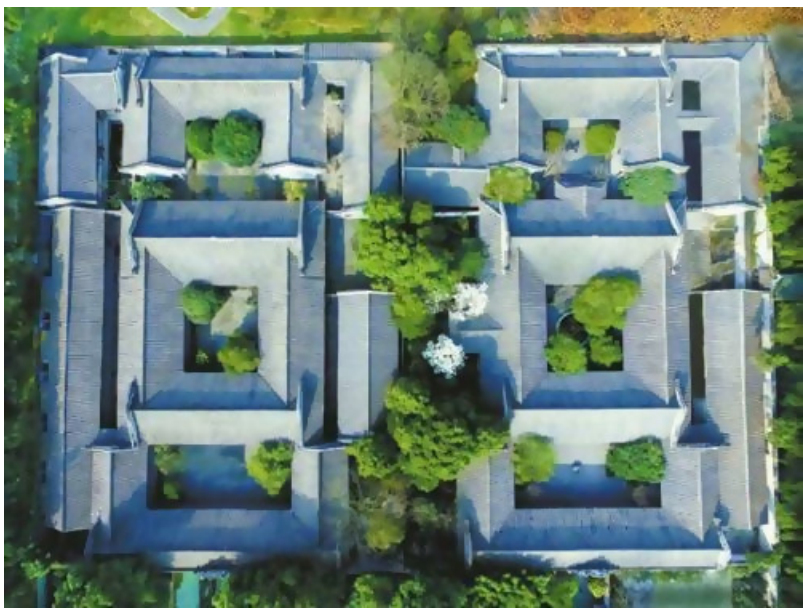

b. The new manor complex.

Fig6. The layout of the two manors showing the courtyards.

In the flexible layout of the old mansion, the family rules of etiquette were hidden in the quadrangle arranged 
in multiple directions, this is the embodiment of Chinese family order and hierarchy. The courtyards are connected, forming a continuous, flexible path, joining the scattered garden spaces, incorporating green space as far as possible in the limited configuration of buildings, and constructing a habitable living space. While the new manor situates the enclosed buildings around the green space under the governance of clear rules of order, the linear path forms a symmetrical axis of the three courtyards, and this garden corridor extends residents' view and enhances the experiential sense of hierarchy in the architectural space. The two layouts of Liu's Manor clearly reflect the importance of the courtyard space in creating a cohesive spatial environment. The patios, both large and small, hold the key to the ventilation of the whole manor and offer bright spots within the innermost spaces. In the courtyards, one can feel the spatial communication between the pastoral environment and the courtyard, linking the living spaces and the nature that surrounds it (Figure 7).

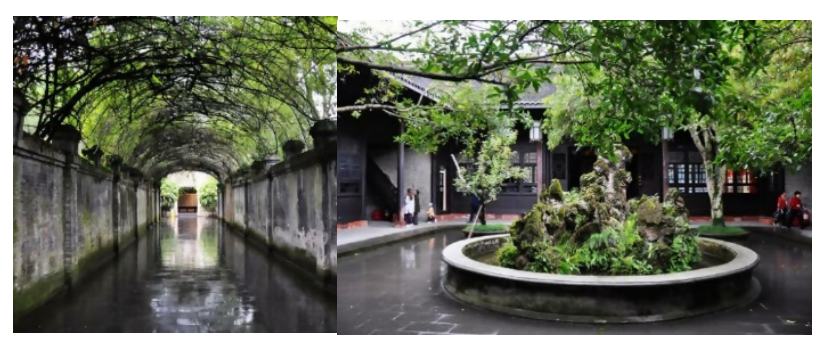

Fig7. The manor's internal spaces.

\subsection{Dispersing to the origin}

\subsubsection{Family origin}

The family system has been integral to traditional Chinese culture since ancient times, especially in the immigrant society of western Sichuan, in which the inheritance of family culture is reflected. According to Liu's Clan Genealogy, Liu's ancestors, Yingliang Liu, emigrated to Sichuan from Huizhou, Anhui Province, in the early Qing Dynasty and were the first settlers in Dayi, laying the foundation for the development of the Liu clan. As recorded in the Great Bulletin in 1949, Wencai Liu is noted as a famous landowner in Sichuan Province, possessing about 800 hectares in farm fields, spread across Dayi and 11 counties in western Sichuan. Furthermore, the article notes numerous properties, including 13 mansions, scattered across Wenjiang, Chongqing, Chengdu, Dayi, and Ya'an. Nevertheless, the manor in Dayi was said to be Wencai Liu's favorite - clearly determined since it was the origin of the family establishment. Liu's manor is divided into two settlements, about 300 meters apart from the north and the south, and comprises five manors built by Wencai Liu and his brothers, which also form part of Liu's ancestral residence. The settlement expansion modeancestral residence, the old mansion, and the new residence-embodies the inheritance and development of clan culture. Liu's ancestral residence is the origin of the manor, and the subsequent construction of the old mansion on the periphery reflects the consolidation and support of the family strength, while the new mansion, as a spatially independent entity, has fully outgrown the power of its family. Thus, the whole history of the family is imprinted in the very construction of the manor complex. The diasporic community, formed by the manor as a whole, highlights the fundamental idea of returning to the origin of the clan, which is the most important conception for the construction of the small-family living environment.

\subsubsection{Agricultural origin}

In addition to the family's origin, the cultural heritage of farming in the Western Sichuan Plain unites the diasporic culture as a whole. The construction of a private estate should form a microcosm of the local social environment so that the sentiment of the family can be amplified into the whole living environment and establish a harmonious sense of Regional Environmental Ethics.

Although Liu's Manor is considered to be the most luxurious private manor in western Sichuan, it is not entirely out of the origin land sentiment of agriculture contained in the dwellings. The ancestral residence in Liu's Manor represents the primitive and plain residential style of western Sichuan, with typical sections for farm production and residential living, including a brewery, dam, vegetable field, and pig pen, which is an authentic portrayal of farming culture in the Western Sichuan Plain (Figure 8). Subsequently, the old mansion and the new mansion expanded one after the other, and are similar to the ancestral residence in terms of space, color, and material. The farming culture is hidden in the landscaping, which preserves the original conception of environmental construction and simultaneously improves the artistic quality of space. In terms of regional pattern, the manor's architecture shows that attention has been paid to a sense of harmony with the surrounding environment. The environmental choices and adaptability of the manor are consistent with the simplicity of a common farmhouse, thus eliminating the class difference in the living environment pattern and appearing as a somewhat symbiotic form attached to the land. The supremacy of the land is reflected in the atmosphere of the microcosmic environmental image to the view of the whole regional environment.

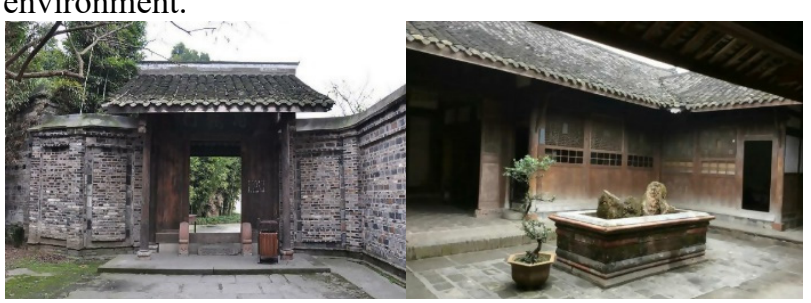

Fig8. The simplicity of Liu's ancestral residence.

\subsection{The integration of classical styles}

The evolution of the residential environment in the Western Sichuan Plain is a turbulent and extraordinary process, which has also determined its regional inclusiveness, not only of immigrant culture but also of Western culture. Combining architectural characteristics of China and the West, as well as those of Sichuan and Anhui, Liu's Manor captures the essence of the region and 
becomes the classical form of rural residential space construction in the Western Sichuan Plain.

\subsubsection{The integration of the traditional building styles of Sichuan and Anhui}

Liu's ancestral residence was the earliest of all the manor buildings and follows the typical style of column-and-tie construction in western Sichuan. Since the family of Liu is native to Anhui, where the traditional architecture style is distinctive from Sichuan, so the later mansions in the manor complex have very obvious features of the Huistyle, the original tone of hui-style architecture is a harmonious combination of white walls, black tiles, special Ma Tau walls, brick, wood and stone carvings, as well as laminated courtyards, high ridges and cornice, winding corridors and pavilions. While in the manor, the features mainly embodied in the shape of the wall and the stone and brick carving. The whole estate was surrounded by brick walls more than six meters high, and the bluegray brick joints were decorated with white wire, giving the manor a sense of a rustic but dignified image in the outside environment. The inner courtyard of the manor is primarily enclosed by the smooth and round "Ma Tau Wall" (Figure 9), which brings a different style to the manor. In addition, the body of the manor buildings remain styled in a wooden column-and-tie structure, with a brick and wood outer corridor, which is a typical southern feature. In such a framework, the two regional styles may have a clear contrast, but share a simple and elegant tone, which is the result of a combination of adaptive choices. The two different cultures are preserved by the most distinct or core characteristics, reconciled at the subordinate and secondary aspects of culture, which eventually transforms the geographic differentiation into spatial dominance.

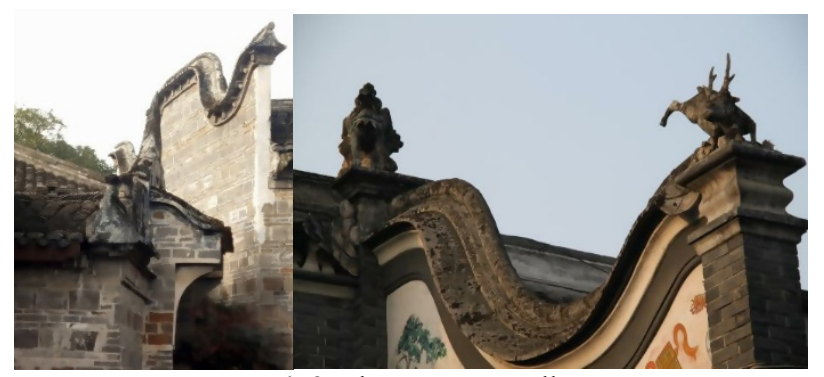

Fig9. The Ma Tau Wall.

\subsubsection{The integration of building styles from China and the West}

The expansive development and construction history of Liu's Manor shows the typical style of the Republic of China in the 1920s and 1930s. However, the combination of Chinese and western characteristics distinguishes the manor from other houses, showing its status, which extends to the family. The gate of the old mansion is a mixture of western Baroque stylistic decoration — columns, arch coupons, domes, and triangular windows-and Chinese stylistic elements, including a calligraphy plaque to enrich the image of the facade. The combination of the two cultures is harmonious. It is not too extravagant but embellished the status of the host (Figure 10).

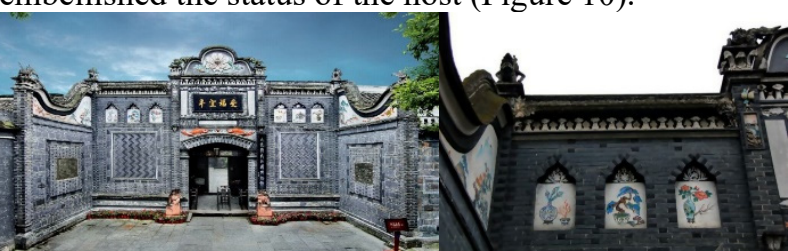

Fig10. The front door and decoration of the old mansion.

Compared to the old mansion, the elegant western style of the new mansion is more obvious. The manor gate has a gothic style, evoking height, slenderness, sharpness and a cold quality. The gate appears perfectly integrated with the blue sky, in the way of a western castle or church. This stylistic choice also reflects the fusion of feudal customs and western religious thought. Furthermore, the formation of the architectural form and style is also a result of the fusion of technology from the cultural background, itself (Figure 11).

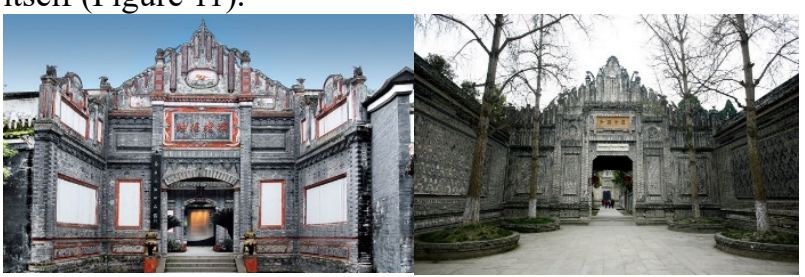

Fig11. Gate modeling showing the combination of Chinese and western stylistic elements.

Currently, the architectural style of the manor is not only a symbol of status, but its environmental aesthetic value also enhances the quality of life of the rural society that surrounds it and enriches the image of the rural environment. The combination of Chinese and western elements in Liu's manor was not exaggerated or deformed but designed to respect the difference in culture between China and the west. In some sense, the design evokes a new classical style by drawing from these original classical styles. By incorporating a limited number of western elements within the greater skeleton of Chinese architecture, the contrast of cultural symbols reflects the residents' ethics in the order of the development of regional space and cultural origin, that is all innovation and integration beyond the region should be carried out around the local original environment, so as to ensures the consistency of the macroscopic spirit of the rural human settlement environment in the West Sichuan Plain.

\section{Conclusion}

Residences can be understood as encompassing a microcosm of the residential environment within a macrocosm of regional pattern orientation. As the largest landlord manor in western Sichuan, Liu's Manor's special economic and cultural background endows it with a high level of environmental selection goal.

Liu's Manor originates from the living environment of rural people in the western Sichuan plain. It gathers in an ideal environment, dispersing to the origin, and the integration of classical styles. The overall settlement form, spatial atmosphere, and architectural style are closely linked to the macrocosmic pattern. This mode of construction preserves the collective memory of the western Sichuan, optimizes and extends the environmental 
texture, location, layout, and cultural elements. Furthermore, the construction forms a personalized integration with the similarity and continuity of the original environment, refining the core concept of the regional environment construction and contributing to the goal of healthy and sustainable development. The analysis of its microcosmic environment, the order formed between its environmental characteristics, and the macrocosmic natural pattern of the Western Sichuan Plain reveals that the owner's selection and integration of regional elements used in Liu's Manor reflect optimal ethical choices. The manor, in terms of settlement environment, family, society, and cultural integration, displays a sense of ideology and virtuosity superior to the general form of living environments in the Western Sichuan Plain.

With increasing attention paid to the construction of the rural residential environment, implementation of the rural revitalization plan needs not only to adapt to local conditions but also to draw on experience to the fullest possibility. In the upsurge of rural construction, Chengdu has listed the restoration of Linpan as a key project. As a typical demonstration of a rural living environment in western Sichuan, the Liu's Manor embodies the origin and integration of the region, setting a standard for the planning and construction of rural settlements. Therefore, the interpretation of the environmental ethical features conveyed by historical architectural classics, like Liu's Manor, explores the harmony and mutually beneficial integration of individuality and commonness, and microcosmic and macrocosmic, so as to provide a reference for the sustainable development of regional human settlements.

The continuously evolving human settlement environment has both a past and a present, the rural human settlement environment needs to establish a reasonable developmental order. As the goal of environmental construction, quantitative changes caused by man's innovation in the living environment should not be separated from the actual situation, leading to the absence of collective memory and disturbing the order of development. The purpose of the present research, on the environmental ethics of historic human settlements, aims to inject collective memory and spirit into the fast-paced trend of thought in construction. Rural construction is rapidly developing, so we should make appropriate use of the history of building in local conditions, develop village settlements that accord with the ethics of the regional human settlement environment, and maintain it. This is essential because this approach to understanding human settlements is helpful to the continuous and stable development of the overall regional environmental order in the time dimension, and can provide a reference for the construction of the rural human settlements environment system, holistically, in the future and contribute to the rejuvenation of the nation.

\section{References}

1. X. Wang, X. Hou, Evolution and Modality of Fortified Lord-Manor Settlements[J]. Journal of
Qingdao University of Technology, 31(03), 6-11 (2010).

2. Y.F. Chen, Ethics of human settlements and greenness of building materials $[\mathrm{J}]$. Jiangxi building materials, $14,35+39(2010)$.

3. S.Y. Wu, Application of Environmental Ethics in Tourism Planning[J]. Northern literature (second half month), 02, 228 (2012).

4. W.M. Li, X.Y. Ye, W.F. Qi, A Review of the Development of Human Settlements Theory and practice at Home and abroad[J]. Journal of Zhejiang University (Science Edition), 02,205-211(2000).

5. Y.X. Fan, A review of the present situation of environmental and ecological ethics in China[J]. Environmental Science \& Technology, 09,108-111 (2007).

6. P.C. Li, J.W. Zhou, The theoretical Origin of Environmental Ethics in the West[J]. Journal of Social Science of Hunan Normal University, 06,22-29+37 (2002).

7. Y. Feng, Research on modern rural construction model based on traditional environmental ethics[D]. Kunming University of Technology, (2012). 